\title{
Research on the Impact of High-speed Railway on the Industrial Structure Change and Carbon Emission of Chengdu-Chongqing Urban Agglomeration
}

\author{
Ge Jingzhao ${ }^{1, *}$ \\ ${ }^{1}$ Beijing Jiaotong University, School of Economics and Management, Beijing, China
}

\begin{abstract}
This paper selects the panel data of 16 cities in the Chengdu-Chongqing urban agglomeration from 2005 to 2015, and uses differences-in-differences model to analyze the impact of high-speed railways on the industrial structure and Carbon Emission of the Chengdu-Chongqing urban agglomeration. The results show that the construction of high-speed rail has reduced the proportion of the secondary industry and has a significant role in promoting the development of the tertiary industry. Among them, fixed asset investment increased the proportion of the secondary industry and the tertiary industry, and technology investment decreased the proportion of the secondary industry, and increased the proportion of the tertiary industry, indicating that the improvement of the level of informatization is helpful to the development of the tertiary industry. It is proposed that a more reasonable evaluation system for the economic effects of highspeed rail should be established. At the same time, the impact of high-speed rail construction on the industrial structure is different. When formulating policies, we must not only weigh the spillover effect and siphon effect of high-speed rail, but also give full play to the carbon reduction effect of economic agglomeration.
\end{abstract}

\section{Introduction}

The construction of transportation infrastructure has a profound impact on the speed and quality of economic development. An accurate understanding of the impact of transportation on economic activities is conducive to a deep understanding of the laws of spatial structure and industrial layout. Our country has a vast land and abundant resources, a large population, and a wealth of total resources, but there are problems such as low per capita possession, uneven distribution of resources, and obvious regional differences. The railway transportation network has achieved a leap from south to north and east to west, which to a certain extent integrates my country's land and land space into one, improves the spatial structure of the economy, and promotes the coordinated development of the country. The railway has become the first choice for people to travel because of its fast running speed, large transportation volume, and low cost. Along with economic development, technological progress, and improvement of residents' living standards, people's requirements for means of transportation have gradually increased. The high-speed railway greatly reduces the time and space distance between cities along the line, extends the travel radius of passengers within a period of time, or saves travel time in a certain area. It is of far-reaching significance to the improvement of regional transportation accessibility, economic and trade exchanges, and the adjustment of industrial structure.
The opening and operation of high-speed rail has promoted the adjustment and transfer of industries in various regions, and to a large extent, it will gradually shorten the economic gap between cities; it will promote the development of the real estate industry, give people more choices in employment, and allow people to choose different living environments; local characteristic tourism will attract more tourists and drive the development of the tertiary industry in local region; promote the rapid flow of labor between regions, urban and rural areas, especially talents, information and other elements. The high-speed railway industry chain is very long, which can drive the optimization and upgrading of related industrial structures. ${ }^{[1]}$

At present, high-speed rail has covered major cities in my country, and convenient transportation has promoted the development of business and trade activities. Frequent exchanges have made the connections between cities closer and strengthened the trend of regional economic integration. Urban agglomerations have become the basic organization for the coordinated development of regional economies in our country. The competition and cooperation between urban agglomerations have made the social connections between regions of different scales and between cities within regions more complex and close. In this context, the construction and operation of high-speed rail have an important positive effect on the acceleration of the process of urban integration, the impact of the economic

* Corresponding author: jzge2021@163.com 
development and industrial structure of the cities within the urban agglomeration, and the adjustment of the functions of the cities in the urban agglomeration. ${ }^{[2]}$

The Chengdu-Chongqing urban agglomeration is a population and economic agglomeration area with the most suitable geographical location, the largest scale, and the closest connection to the national and world economy in western China. It is one of the three major population and economic core areas of the Yangtze River Economic Belt in my country. It is a hub that connects the China-Singapore International Economic Corridor and a convenient hub for the New Eurasian Continental Bridge International Economic Corridor. It has superior development conditions and huge development potential, which is of great significance to promoting the high-quality development of the western economy. The opening of high-speed railways has an important impact on the changes in the industrial structure of the Chengdu-Chongqing urban agglomeration.

Regarding the impact of high-speed rail on the region, many domestic scholars have used econometric models to conduct in-depth research on it. Jin Feng (2021) established a centripetal fractal model and a correlation fractal model to analyze the changes in the fractal characteristics of the traffic network spatial structure of the Northeast urban agglomeration, and concluded that when Shenyang is the center, the spatial density of the Northeast urban agglomeration changes more evenly from the center to the periphery. In the era of high-speed railway, the spatial agglomeration and distribution of the transportation network of the northeast urban agglomeration are more significant, and the spatial relevance is stronger. ${ }^{[3]}$ Ren \& Lin (2021) used the panel data of 277 cities in my country from 2004 to 2016 to expand the Cobb Douglas function and build a spatial model to investigate the impact of high-speed rail on urban economic growth. The article concludes that from the perspective of the scale of cities across the country, the opening of high-speed rail drives the local GDP growth by 1.96 percentage points, while promoting the economic growth of other cities; in terms of the cumulative effect of time, it is demonstrated that for each year of increase in the opening time of high-speed rail, local GDP growth is 0.6 percentage points. At the same time, it also concluded that there is a big gap between the economic benefits of high-speed railway construction and heterogeneous cities. For every year of high-speed railway operation, the GDP of central cities will increase by $1.3 \%$, while the GDP of peripheral cities will only increase by $0.3 \%$. It further discusses the impact mechanism of high-speed rail on consumption from the spatial dimension, and finds that the construction of high-speed rail promotes consumption growth and drives the consumption economy of surrounding cities. ${ }^{[4]} \mathrm{Cen}$ Jinhao (2020) analyzed the impact of the opening and operation of high-speed rail on the economic integration of the high-speed rail development in the Yangtze River Delta, and found that the opening of high-speed rail has less impact on the economic integration of core cities than in peripheral cities, and has less impact on large cities than small and medium-sized cities. ${ }^{[5]}$ Zhang
Jiageng (2020) took the Zheng-Wu high-speed rail's impact on the industrial structure and competitiveness of the areas along the line as the research object, and found that after the opening of the Zheng-Wu high-speed rail, the spatial competitiveness of the first and second industries in the areas along the line tends to be balanced, and at the same time, the spatial competitiveness of the tertiary industry in the areas along the route tends to be unbalanced ${ }^{[6]} \mathrm{Li}$ Jinyu (2019) analyzed the impact of the opening of the high-speed rail on the urban industrial structure based on the Guangdong-Hong Kong-Macao Greater Bay Area, and found that the opening of the high-speed rail significantly promotes the rationalization of the overall industrial structure, that is, the upgrade; the operation of the high-speed rail has a significant positive effect on the development of the manufacturing industry; The opening of the high-speed rail has played a significant role in promoting the tertiary industry; the opening of the high-speed rail has also promoted the development of the accommodation and catering industry, financial industry and real estate in the tertiary industry. ${ }^{[7]}$ Peng Xue (2017) analyzed the changes in the industrial structure of cities along the BeijingGuangzhou high-speed railway, and found that the tertiary industry in small and medium-sized cities has developed rapidly after the opening of the high-speed rail, and economic contribution and competitiveness advantages have been improved. In large-scale cities, the secondary industry develops rapidly, and the competitive advantage is higher than that of medium-scale cities. ${ }^{[8]}$

\section{Model construction}

This article uses differences-in-differences method, which can largely avoid endogenous problems: relative to microeconomic entities, policies are generally exogenous, so there is no adverse causality problem. In addition, the use of fixed-effects estimation also alleviates the problem of missing variables to a certain extent. The traditional method of evaluating policy effects is mainly through setting a dummy variable of whether the policy occurs or not and then performing regression. In comparison, the model setting of differences-in-differences method is more scientific and can more accurately estimate the policy effect.

$Y_{i t}=\beta_{0}+\beta_{1}$ rail $_{i t}+\beta_{2} t_{i t}+\beta_{3}$ rail $_{i t} * t_{i t}+X_{i t}+\varepsilon_{i t}$

The data used in this article are from the "China City Statistical Yearbook" and "Chongqing City Statistical Yearbook", and the data of 16 cities in the ChengduChongqing urban agglomeration from 2005 to 2015 are selected. The Cheng-mian-le intercity and ChengduChongqing high-speed railways started construction in 2009 and 2010 respectively, and both were under construction in 2010. Therefore, for convenience, the 2009 is ignored and divide the entire sample interval into two periods of 2005-2009 and 2010-2015, and use $t$ as the time dummy variable. When it is in the period of $2005-2009, \mathrm{t}$ is 0 ; when it is in $2010-2015, \mathrm{t}$ is 1 . Define trail as rail ${ }_{\mathrm{i}}{ }^{*} \mathrm{t}_{\mathrm{it}}$, and it is 1 after high-speed rail is 
opened and running, otherwise it is $0 . \varepsilon_{\text {it }}$ represents the random disturbance term.

Where $\mathrm{i}$ and $\mathrm{t}$ represent the $\mathrm{t}$-th year of the $\mathrm{i}$-th city; The explained variable $\mathrm{Y}_{\text {it }}$ represents the impact of highspeed rail on the industrial structure of the ChengduChongqing urban agglomeration, which is measured by the proportion of the secondary industry in GDP (S2) and the proportion of tertiary industry in GDP (S3). rail ${ }_{\text {it }}$ is a dummy variable of the high-speed rail construction status. When it belongs to the cities where two highspeed rails are under construction or operation, the rail value is 1 , which is the experimental group. The cities in the experimental group mainly include eight cities including Chengdu, Chongqing, Deyang, Ziyang, Meishan, Leshan, Mianyang, and Neijiang; On the contrary, if the city does not belong to the construction of two high-speed rails, the rail value is 0 , which is the control group. The cities in the control group include eight cities including Zigong, Dazhou, Ya'an, Luzhou, Suining, Nanchong, Yibin, and Guang'an.

The explanation about the variables is as follows:

S2 (The proportion of the secondary industry in GDP): Secondary industry output value/city GDP; S3(The proportion of tertiary industry in GDP): Tertiary industry output value/city GDP; t(Time factor): Time dummy variable. The value of Chengmianle intercity and Chengdu-Chongqing high-speed railway is 1 when construction starts in 2010, and 0 before 2010; rail(Regional factors): Regional factors, experimental group includes Chengdu, Chongqing, Deyang, Mianyang, Neijiang, Ziyang, Meishan, Leshan, rail=1, control group includes Zigong, Luzhou, Suining, Nanchong, Yibin, Guang'an, Dazhou, Ya'an, rail=0; trail(High-speed rail factor):High-speed rail factor, the high-speed rail is open and running, it is 1 , otherwise it is 0 ; retail(Retail sales of consumer goods): Retail sales of consumer goods in the city; land(Land area of built-up area): In the urban administrative area, there are actually areas where development and construction, municipal public facilities and public facilities are basically equipped; edu(Education investment): Education expenditure/ financial expenditure; $\mathrm{rd}($ Technology investment): Education expenditure/financial expenditure; asset(Total investment in fixed assets): Total investment in fixed assets of the city.

\section{Results}

Table 1 shows the impact of high-speed rail and control variables on the secondary and tertiary industries.

Table 1. The impact of the opening of high-speed rail on the

\begin{tabular}{|c|c|c|}
\multicolumn{3}{|c}{ urban industrial structure } \\
\hline Variables & S2 & S3 \\
\hline \multirow{2}{*}{ trail } & $\begin{array}{c}-2.029^{*} \\
(-1.72)\end{array}$ & $\begin{array}{c}0.068^{* *} \\
(1.07)\end{array}$ \\
\hline \multirow{2}{*}{$\mathrm{t}$} & $\begin{array}{c}12.062^{* * *} \\
(14.85)\end{array}$ & $\begin{array}{c}-3.357^{* * *} \\
(-4.75)\end{array}$ \\
\hline \multirow{2}{*}{ asset } & $\begin{array}{c}4.73 \mathrm{e}-08 \\
(0.51)\end{array}$ & $\begin{array}{c}6.07 \mathrm{e}-08 \\
(0.76)\end{array}$ \\
\hline \multirow{2}{*}{ retail } & $\begin{array}{c}-5.15 \mathrm{e}-07^{* * *} \\
(-3.28)\end{array}$ & $\begin{array}{c}3.38 \mathrm{e}-07^{* *} \\
(2.47)\end{array}$ \\
\hline
\end{tabular}

\begin{tabular}{|c|c|c|}
\hline land & $\begin{array}{c}0.178^{*} \\
(1.74)\end{array}$ & $\begin{array}{c}-0.021^{* *} \\
(-2.38)\end{array}$ \\
\hline \multirow{2}{*}{ edu } & $\begin{array}{c}0.517 \\
(0.06)\end{array}$ & $\begin{array}{c}-0.765 \\
(-0.10)\end{array}$ \\
\hline \multirow{2}{*}{$\mathrm{rd}$} & $\begin{array}{c}-0.538 \\
(-0.07)\end{array}$ & $\begin{array}{c}0.964 \\
(0.15)\end{array}$ \\
\hline \multirow{2}{*}{$\mathrm{C}$} & $43.354^{* * *}$ & $34.196^{* * *}$ \\
& $(27.41)$ & $(24.84)$ \\
\hline $\mathrm{N}$ & 271 & 271 \\
\hline $\mathrm{R}^{2}$ & 0.5709 & 0.1675 \\
\hline
\end{tabular}

The impact coefficient of high-speed rail on the secondary industry is -2.029 , which is significant at the $10 \%$ level, and the impact coefficient of high-speed rail on the tertiary industry is 0.068 , which is significant at the $5 \%$ level, indicating that the opening of high-speed rail reduces the proportion of the secondary industry and increases the proportion of the tertiary industry. At the same time, the impact coefficients of total retail sales of social goods and technology expenditures on the secondary industry are $-5.15 \mathrm{e}-07$ and -0.538 , respectively, indicating that these have a negative effect on the secondary industry. The fixed asset investment, land built-up area, and education investment have an impact coefficient of $4.73 \mathrm{e}-08,0.178$, and 0.517 on the secondary industry, respectively, indicating that these have a positive effect on the secondary industry. The influence coefficients of the area of built-up land and education expenditure on the tertiary industry are -0.021 and -0.765 , respectively, indicating that these have a negative effect on the tertiary industry. The impact coefficients of fixed asset investment, total retail sales of social goods, and technology investment on the tertiary industry are $6.07 \mathrm{e}-08,3.38 \mathrm{e}-07$, and 0.964 , respectively, indicating that these have a positive effect on the tertiary industry.

\section{Conclusion}

This paper uses the panel data of 16 prefecture-level cities in Sichuan and Chongqing from 2005 to 2015 to study the impact of the opening of high-speed rail on the industrial structure of the Chengdu-Chongqing urban agglomeration. The empirical research results show that the opening of the high-speed rail has reduced the proportion of the secondary industry and has a significant role in promoting the development of the tertiary industry.

Among them, fixed asset investment increased the proportion of the secondary industry and the tertiary industry; science and technology investment has reduced the proportion of the secondary industry and increased the proportion of the tertiary industry, indicating that the improvement of the level of informatization is conducive to the development of the tertiary industry; at the same time, the area of built-up land has increased the proportion of the secondary industry and decreased the proportion of the tertiary industry; the total retail sales of social goods has reduced the proportion of the secondary industry and increased the proportion of the tertiary industry, indicating that most of these funds are currently 
invested in the tertiary industry; education investment has increased the proportion of the secondary industry and decreased the proportion of the tertiary industry. The opening of high-speed rail does have different impacts on the industrial structure of different cities. It cannot be generalized that high-speed rail has promoted the optimization of regional industrial structure. Instead, the impact of high-speed rail should be investigated in light of the city's own characteristics, and the actual role of high-speed rail should be studied by integrating the city's adsorption capacity, resource integration capacity, geographic location, policy system and other factors.

\section{Policy recommendations}

1. Cooperate with corresponding policies to promote economic development. The opening of the high-speed rail has different impacts on the industrial structure. The industrial structure should be adjusted in accordance with specific conditions and corresponding policies. This not only facilitates people's travel, but also promotes the optimization of local industrial structure and economic development.

2. Adjust the industrial structure based on corresponding indicators. The opening of the high-speed rail has reduced the proportion of the secondary industry and has a significant promotion effect on the development of the tertiary industry. The industrial structure must be adjusted according to the corresponding indicators.

3. Establish a more reasonable evaluation system for the economic effects of high-speed rail. From the perspective of changes in the industrial structure, a complete high-speed rail economic effect evaluation system should be established. It can not only realize the rational use of high-speed rail and maximize social welfare, but also give play to the influence of high-speed rail on urban agglomerations, adjust the industrial structure, and promote economic development. China's high-speed rail economic effect evaluation system is not complete, and is quite different from foreign research and evaluation systems. It can be appropriately combined to establish a more reasonable high-speed rail economic effect evaluation system. ${ }^{[9]}$

4. As the pace of life in modern society continues to accelerate, high-speed rail is not only an important part of measuring whether a city has a complete modern infrastructure system, but also an important urban transportation method indispensable for modern cities. Therefore, each city should promote the construction of high-speed rail in each city on the basis of fully considering the actual situation in each region. In turn, it can meet people's diversified travel needs, improve traffic accessibility, shorten time and space distance, and provide new development opportunities for economic development and industrial structure optimization and upgrading in various regions. ${ }^{[10]}$

5. When formulating policies, we must not only weigh the spillover effect and siphon effect of highspeed rail, but also give full play to the carbon reduction effect of economic agglomeration. Therefore, local governments should give full play to the advantages brought by high-speed rail and formulate a series of preferential policies to attract the concentration of elements in surrounding non-high-speed rail cities. Economic agglomeration will further promote the reduction of carbon emission intensity by realizing economies of scope and strengthening the effects of environmental regulation, and realize the development of low-carbon economy. In the process of high-speed rail construction, cities need to formulate long-term urban planning and urban strategies, and need to have a clear city positioning, and carry out the construction and planning of high-speed rail new districts within the economic and environmental carrying capacity of each city.

\section{References}

1. T. Guo, Southwestern University of Finance and Economics, Research on the Impact of High-speed Rail on the Industrial Structure Changes of Chengdu-Chongqing Urban Agglomeration (2019).

2. X.P. Chao, L. Zeng, Housing Technology, The impact of high-speed rail on the development of China's urban agglomerations: Taking the Central Plains urban agglomeration as an example, 40:24-29 (2020).

3. F. Jin, China Railway, Research on the Spatial Structure Characteristics of the Traffic Network of the Northeast Urban Agglomeration Influenced by the Harbin-Dalian High-speed Railway, 02:95-100 (2020).

4. T.X. Ren, J.Y. Lin, Mathematical Statistics and Management, Research on the Spatial Spillover Effect of the Opening of High Speed Rail on Urban Economic Growth_—Based on Evidence from 277 Core Cities, 20 :1-16 (2021).

5. J.H. Cen, Jilin University, Research on the impact of high-speed rail on regional economic integration (2020).

6. J.G. Zhang, Nanjing University, Research on the Impact of Zhengwu High-speed Railway on the Industrial Structure and Competitiveness of the Areas Along the Line (2020).

7. J.Y. Lin, Harbin Institute of Technology, An Empirical Study on the Impact of the Opening of High-speed Rail on Urban Industrial Structure (2019).

8. X. Peng, Beijing Jiaotong University, Analysis of Industrial Structure Changes in Cities Along the High-speed Railway (2017).

9. F.Y. Chen, Asting Model, Journal of Jingdezhen University, Research on the Impact of Anhui Highspeed Railway on Industrial Structure-Based on Grey Forec, 35:69-73 (2020).

10. K. Shao, W.P. Zhu, D. Liu, Southern Economy, Can High Speed Rail Promote the Upgrading of Industrial Structure: Based on the Perspective of Resource Redistribution., 02:56-72 (2020). 